\title{
Imaging and Ion-Beam Milling of Biological Specimens with the Helium-Ion Microscope
}

\author{
Matthias Schmidt ${ }^{1}$, Chaturanga Bandara ${ }^{1}$, Marc Tamisier ${ }^{1}$, Ilari Maasilta ${ }^{2}$ and James Byrne ${ }^{3}$ \\ ${ }^{1}$ Helmholtz-Centre for Environmental Research GmbH - UFZ, Leipzig, Sachsen, Germany, ${ }^{2}$ University of \\ Jyväskylä, Jyväskylä, Finland, ${ }^{3}$ University of Bristol, Bristol, England, United Kingdom
}

The last decade has seen several spectacular studies focusing on medical research, plants and small animals, microbiology and geomicrobiology using helium-ion microscopy (HIM) as a tool for high-resolution imaging and, to a lesser extent, nano-fabrication of biological objects [1]. HIM in many aspects resembles scanning electron microscopy (SEM), however, its high surface sensitivity, the possibility to image insulating specimens without having to coat them with a conductive material and the capability of gentle milling with a beam of highly focused light ions ( $\mathrm{He}$ or $\mathrm{Ne}$ ) render the technique preferable over SEM for many applications. In terms of resolution, HIM is closer to that of a standard, non-aberration-corrected transmission electron microscope (TEM) which enables imaging of some the tiniest structures in nature without thin lamella preparation. A recent, particularly spectacular example of this was the first HIM images of SARS-CoV2 viruses [2].

In the first part of this presentation we give a brief overview of the available literature on HIM imaging of biological samples and review some highlights that demonstrate the particular capabilities that the technique has to offer. We touch on the importance of sample preparation on the obtained micrographs and discuss whether HIM could benefit from well-established techniques used in electron microscopy, further outlining a protocol which we have developed to prepare bacterial samples [3]. It has several parameters to adapt to different experimental requirements and was successfully tested with different bacterial strains. We will briefly elaborate on the different contrast mechanisms that can be used in HIM provided the availability of suitable detectors. So far, bio-imaging has mostly relied on secondary electron detection, however, detecting transmitted or back-scattered ions, iono-luminescense or employing one of the recently developed and commercialised secondary ion mass spectrometers for the HIM is extremely promising for future life-science studies For example, these new detectors could help greatly advance the investigation of the interactions of nano-particles with cells or tissues.

In the second part of the presentation we will provide a brief overview of the application of HIM for ion-beam milling of biological objects including nematodes, bacteria, bacteriophages and dragonfly wings. Thereafter, we present some of our latest results on ion-beam milling of insect wings, microbes and microbial-induced corrosion crusts. We observed that successful milling strongly depends on controlling the heat damage of the sample due to the temperature increase under the beam [4]. Therefore, once the choice of the ion and its landing energy is made, the combination of beam current, dwell-time and spacing has to be optimised for the particular material lest it deteriorate or even melt. We systematically determined such a parameter set for insect wings [5] consisting mostly of chitin and lipids and applied SRIM simulations [6] and physical arguments to estimate the local temperature. With these optimised parameters it was possible to mill a line of about $35 \mathrm{~nm}$ thickness through the nano-pillars on the wing of a dragonfly.

Overall, in this presentation we can only highlight just some of the exciting studies on bio-imaging with the HIM during the past decade and show a few of our latest results. However, the full potential of the HIM is far from being fully exploited and we hope that this powerful method will establish a firm place amongst the standard characterisation tools for bio-imaging and become more frequently used for nano-fabrication of biological objects during this decade. 


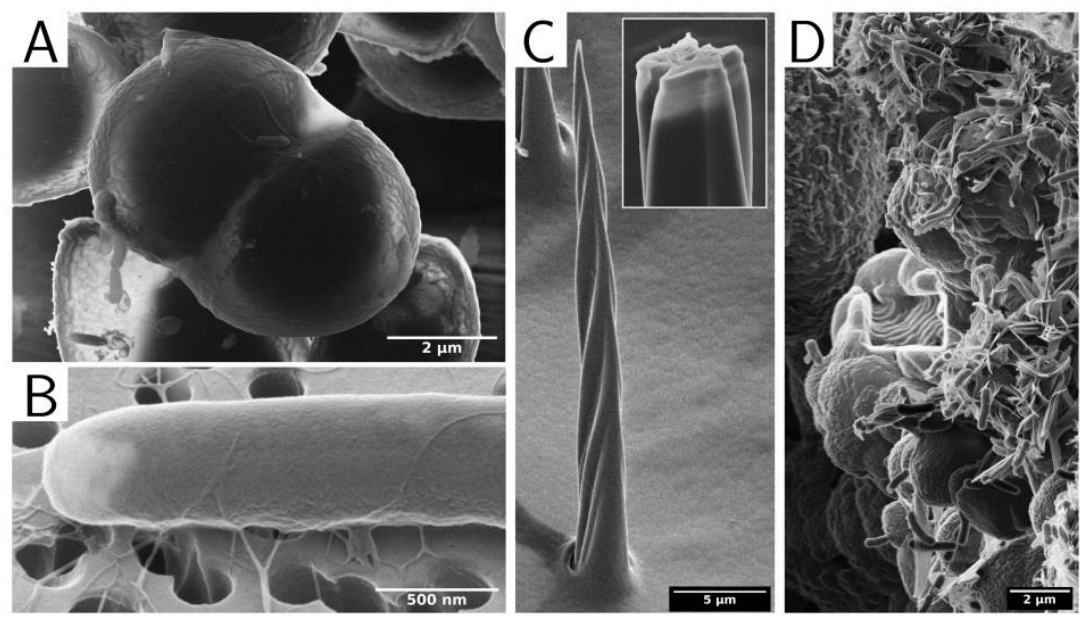

Figure 1. A) HIM images of A) a Chlorella microalgae (sample prepared by J.-H. Moreno-Osorio within the work of [5]) and B) a bacterial sample of Pseudomonas putida with flagella (sample prepared by F. Calabrese and N. Said). Ion-beam milling of C) a wing-hair of a bee (miling carried out by R. Dittrich) and D) into the crust on a microbially corroded steel coupon.

\section{References}

[1] Matthias Schmidt, James Byrne and Ilari Maasilta, Beilstein J. Nanotechnol. 2021, 12, DOI: 10.3762/bjnano.12.1

[2] Natalie Frese, Patrick Schmerer, Martin Wortmann, Matthias Schürmann, Matthias König, Michael Westphal, Friedemann Weber, Holger Sudhoff and Armin Gölzhäuser, Beilstein J. Nanotechnol. 2021, 12, DOI: $10.3762 /$ bjnano.12.13

[3] The authors acknowledge scientific and technical assistance of Federica Calabrese, Niculina Musat, Katja Nerlich, Nedal Said and Hryhoriy Stryhanyuk at Helmholtz - Centre for Environmental Research GmbH UFZ, ProVIS, Leipzig, Germany

[4] A. Wolff, N. Klingner, W. Thompson, Y. Zhou, J. Lin, Y.Y. Peng, J.A.M. Ramshaw and Y. Xiao, J. Microscopy 2018, 272 (1), pp. 47-59, DOI: 10.1111/jmi.12731

[5] The authors are grateful to Robin Dittrich for systematic ion-beam milling of wings collected from bees and dragonflies (no killing of insects, for this study only dead animals were collected) during his internship at Helmholtz - Centre for Environmental Research GmbH - UFZ, ProVIS, Leipzig, Germany.

[6] J.F. Ziegler, J.P. Biersack and M.D. Ziegler 2015 SRIM - the stopping and range of ions in matter. SRIM Company. ISBN: 0-9654207-1-X

[7] Jairo H. Moreno Osorio, Pietro Benettoni, Matthias Schmidt, Hryhoriy Stryhanyuk, Mechthild SchmittJansen, Gabriele Pinto, Antonino Pollio, Luigi Frunzo, Piet N.L. Lens, Hans H. Richnow, Giovanni Esposito and Niculina Musat, FEMS Microbiology Ecology 2019, 95, fiz029, DOI: 10.1093/femsec/fiz029

[8] The authors are thankful for the use of the helium-ion microscope at the Centre for Chemical Microscopy (ProVIS) at UFZ Leipzig, which is supported by European Regional Development Funds (EFRE-Europe funds Saxony) and the Helmholtz Association, and for the use of the helium-ion microscope at University of Jyväskylä, which is funded by the Academy of Finland within the FIRI2014 programme. 